\title{
Adaptive Variable-speed Control for Enhancing the Performance of a Mine Counter-rotating Fan System
}

\author{
Zijian Ai, ${ }^{1,2}$ Guoliang Qin, ${ }^{1}$ Kun-Chieh Wang, ${ }^{2 *}$ and Hao $\mathrm{Gao}^{2}$ \\ ${ }^{1}$ School of Energy and Power Engineering, Xi'an Jiaotong University, Xi'an 710049, Shaanxi Province, China \\ ${ }^{2}$ School of Mechanical and Electronic Engineering, Sanming University, Sanming 365004, Fujian Province, China
}

(Received March 23, 2021; accepted July 16, 2021)

Keywords: mine counter-rotating fan, adaptive speed control, performance enhancement of ventilation

The mine counter-rotating fan (MCRF) is important in tunnel construction and is also widely used for ventilation in mining. The need to improve the performance of MCRF systems has been attracting the interest of researchers. An MCRF is composed of two vane rotors that rotate in opposite directions. We propose a novel adaptive variable-speed (AVS) control method to improve the performance of an MCRF system. The proposed method is based on the concept of adaptively controlling the speed of the second vane rotor according to changes in the working conditions of the whole fan system. To evaluate the performance of our proposed AVS control method, experiments were performed using various sensors to detect pressure and mass flow rates when using our newly designed MCRF system. Results show that, with the proposed AVS control method, the problem of the rotor output torque rising and falling markedly during large and small mass flows, respectively, is overcome. Furthermore, the problems of overload and a low blocking margin are solved. As a result, the overall pressure increase and performance of the whole fan system are improved.

\section{Introduction}

The mine counter-rotating fan (MCRF) is extensively applied to the ventilation of mines and tunnels during their construction. The safety and performance of the MCRF are key factors ensuring the safety of human lives and productivity in the construction industry, respectively. ${ }^{(1-3)}$ The MCRF is composed of two vane rotors, named R1 and R2. These two rotors rotate in opposite directions and each can be mutually used as a guide vane for each other. Owing to their compact structure, large pressure increase, and high performance, MCRFs are considered as effective turbomachinery for ventilation. ${ }^{(4)}$

The performance of an MCRF system is good under specified operation conditions of steady flow and small pressure loss. ${ }^{(5,6)}$ However, most of the time, an MCRF system operates under unspecified conditions, under which its performance deteriorates. ${ }^{(7-9)}$ In the early stage of operation of the system, the wind resistance at the mesh of the pipe inlet is small. The actual fluid discharge is smaller than that according to the theoretical design. Moreover, the pressure increase and performance of whole system are small. In the middle stage of working, the wind

*Corresponding author: e-mail: 20190207@fjsmu.edu.cn https://doi.org/10.18494/SAM.2021.3394 
resistance at the mesh of the pipe approaches the designed pressure increase of the MCRF system. The whole fan system exhibits good performance. In the late stage, the wind resistance at the mesh of the pipe inlet increases again. The actual fluid discharge again becomes smaller than that according to the theoretical design, similarly to that observed in the early stage. In this stage, the load of R2 increases rapidly and will easily cause overload burnout. In the case of overload burnout, the whole fan system enters a surging condition, which is extremely harmful to the system itself as well as to personnel at work. To ensure the safe operation and high performance of an MCRF system, it is necessary to enlarge the surge and blocking margin and adjust the working range. Previous studies indicated that the capacity of MCRFs can be modified by optimizing the design of the impeller structures and the angles of installation. ${ }^{(10-14)}$ Some studies $^{(15-19)}$ found that optimizing the gap between the impeller tips of rotors may significantly improve the efficiency and stability of operation for an MCRF system. This is because the gap size of R1 may affect the occurrence and development of a turbulence trail in the impeller tip region, and thus the flow conditions in the impellers of R2. Furthermore, the size of the axial space between the two rotor centers affects the extent of fluid interaction between R1 and R2. As the axial space increases, flow instability will weaken, but the unsteady characteristic of the fluid flow in both R1 and R2 tends to become consistent. ${ }^{(20-24)}$ To solve the overload problem encountered in R2 under small-flow conditions, a common solution is to adopt different loads (e.g., by reducing the load) or raise the power margin for R2. ${ }^{(25-28)}$ Nevertheless, these measures usually reduce the overall performance for other flow conditions. Some studies showed that adaptively changing the load distribution by adjusting the impeller velocity of the rotor may effectively and optimally enhance the overall performance for all flow conditions. ${ }^{(29-31)}$

Most of the previous studies on enhancing the performance of an MCRF system focused on passively adjusting suitable parameters to fit specific surging or designed working conditions. These methods have various limitations and fail under other undesigned working conditions. Therefore, it is urgently necessary to find a suitable way to adaptively control related parameters for different working conditions.

To meet this need, we propose an adaptive variable-speed (AVS) method that may be used to automatically adjust the operating speed of R2 according to the flow conditions to enhance the overall performance of the MCRF system for the entire working range and also solve the problem of overload in R2 under small-flow conditions.

\section{Problems and Control Methodology}

\subsection{Current problems of MCRF systems}

To address our proposed novel AVS control method for enhancing the overall performance of an MCRF system, we design a small MCRF system with a rated pressure of $2.8 \mathrm{kPa}$ and a mass flow rate of $4.25 \mathrm{~kg} / \mathrm{s}$. The related basic parameters of our designed MCRF system are listed in Table 1. ${ }^{(31)}$ Then, performance tests of our designed MCRF system are carried out using the D-type test method (inlet and outlet test) using Chinese standard GB-1236-2000. The designed MCRF system as well as its test instruments include numerous pressure and power sensors, as 
Table 1

Parameters of the two-stage MCRF system designed by our team.

\begin{tabular}{lcc}
\hline Parameters & First-stage impeller & Second-stage impeller \\
\hline Number of vanes & 12 & 10 \\
Designed speed & $2930 \mathrm{rpm}$ & $-2930 \mathrm{rpm}$ \\
Vane diameter & $558 \mathrm{~mm}$ & $558 \mathrm{~mm}$ \\
Wheel-to-shell ratio & 0.65 & 0.65 \\
Rated power & $11 \mathrm{~kW}$ & $11 \mathrm{~kW}$ \\
Rated efficiency & 0.82 & 0.82 \\
\hline
\end{tabular}
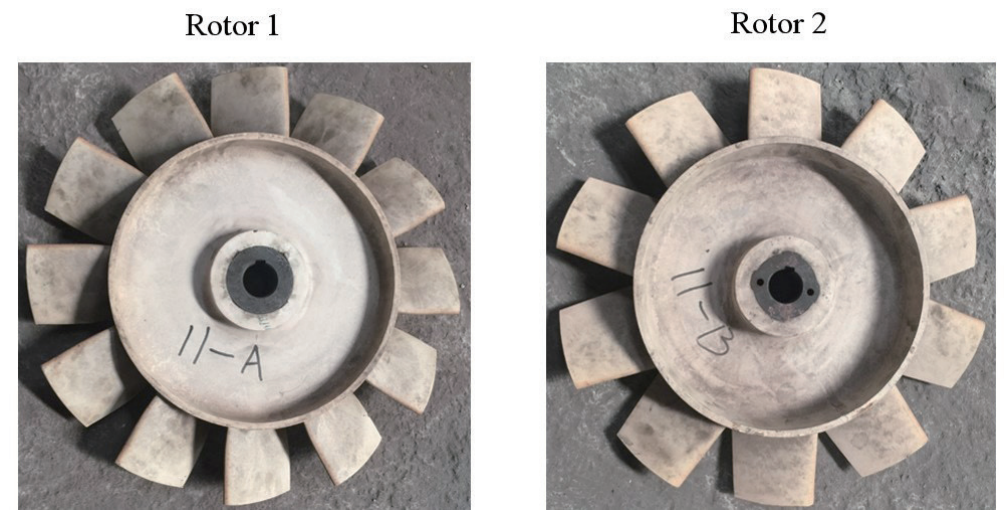

Fig. 1. (Color online) Rotors of two-stage MCRF system.

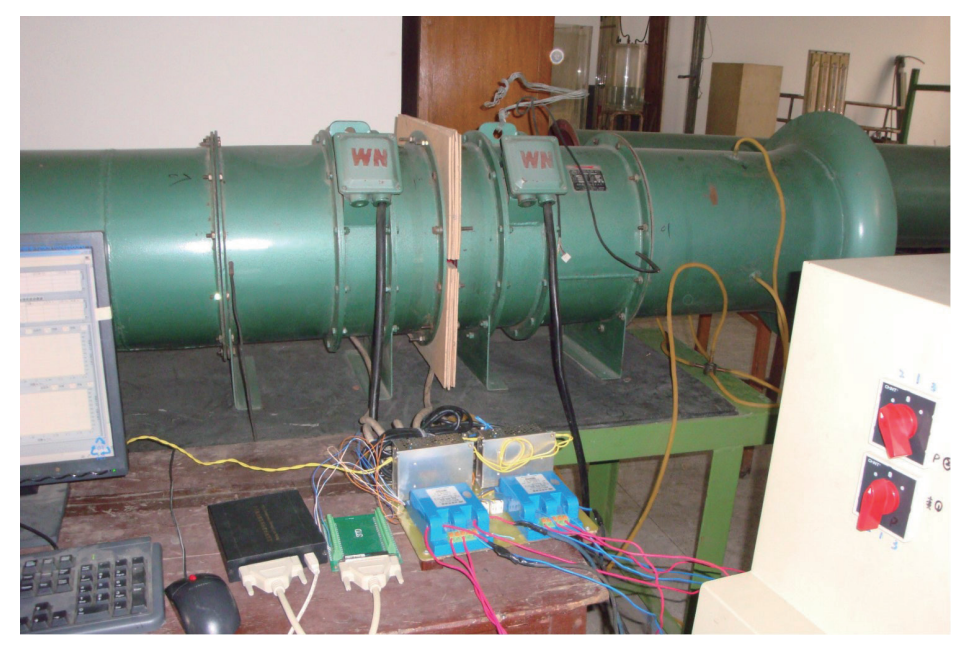

Fig. 2. (Color online) MCRF system and its test device.

shown in Figs. 1 and 2. The measured pressures and torques are used to evaluate the aerodynamic performance of the designed MCRF and the powers of the two rotors in real time for adaptive speed control. The pipe diameters of the inlet and outlet are denoted as $d_{1}$ and $d_{2}$, respectively. The inlet static pressure $p_{1}$ is measured at a point located in the inlet pipe about $2 d_{1}$ in front of the first-stage impeller, and the outlet static pressure $p_{2}$ is measured at a point located in the outlet pipe about $2 d_{2}$ downstream from the second-stage impeller. The frequency converters, 


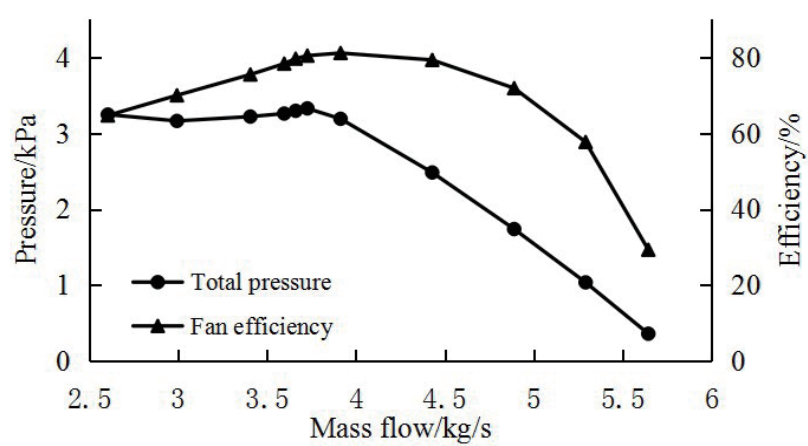

Fig. 3. Variations of pressure and overall fan efficiency with mass flow rate in case of equal-speed operation for R1 and R2.

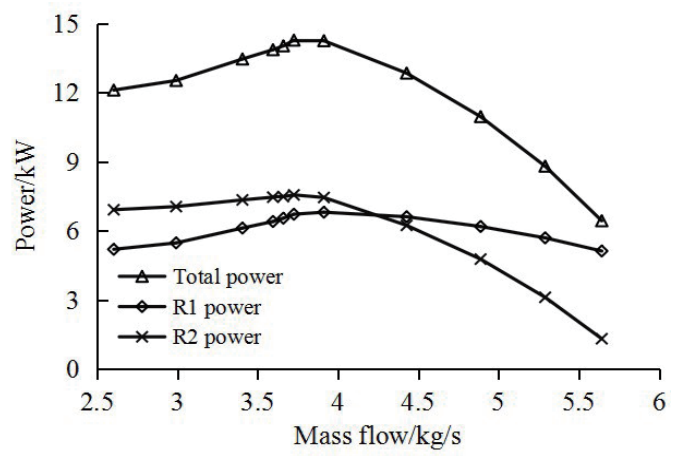

Fig. 4. Power versus mass flow rate for R1 and R2.

which are connected to rotors, are used to monitor the speeds of R1 and R2. The power sensors connected to the rotors are used to measure the input powers of R1 and R2, which are respectively denoted as $N_{1 m}$ and $N_{2 m}$. The shaft powers of the rotors may be derived from their input powers and efficiencies.

The variations of the measured pressure and calculated fan efficiency with the flow rate for $\mathrm{R} 1$ and R2 are shown in Fig. 3, and the variation of the power with the flow rate for R1 and R2 is shown in Fig. 4. In Fig. 3, it can be seen that the pressure and fan efficiency increase slightly and then decrease with increasing mass flow rate. In Fig. 4, it can be seen that the power of R1 has only small variations with the mass flow rate. However, the power of R2 varies markedly with the mass flow rate. Note that a rapid increase in the power of R2 may lead to the overloading of the whole system in the case of a small flow. On the other hand, a rapid decrease in the power of R2 may cause low overall efficiency.

\subsection{Methodology of AVS control for an MCRF system}

\subsubsection{Control principle of AVS method}

The power of R1 is hardly affected by the variation of the flow, and thus its capacity may be kept at a good level. However, the variance of the flow significantly affects the power of R2 and therefore causes large fluctuations in its capacity. The capacities of R1 and R2 are similar and both exhibit good levels under designed working conditions. Nevertheless, their capacities deteriorate and diverge when the working conditions deviate from the designed ones. To solve this problem, we propose a novel control method called the AVS method.

The AVS control method mainly focuses on adjusting the speed of R2. When the working condition changes, we keep the speed of R1 unchanged and, in real time, adjust the speed of R2 while ensuring that both R1 and R2 always have the same power output. That means that the power output of R2 $\left(N_{2}\right)$ is always kept at the same magnitude as that of R1 $\left(N_{1}\right)$. The control loop of AVS is shown in Fig. 5. In the AVS method, we choose $N_{1}$ as the target signal and $N_{2}$ as 
the feedback signal. Through PID control, we may examine the difference between $N_{1}$ and $N_{2}$ in real time and adaptively adjust the speed of R2 $\left(N_{2}\right)$ via a frequency converter to ensure that the power output of R2 is always equivalent to that of R1. Eventually, the goal of operation with equivalent power output for both rotors from instant to instant is reached.

\subsubsection{Control process and hardware of AVS method}

On the basis of the control principle mentioned in Sect. 2.1, we now develop the control process of the AVS method. As shown in Fig. 6, we first measure the input powers of R1 and R2 in real time via power sensors 1 and 2, respectively. Second, we send these powers to a data collector through connecting terminals. These message data are converted from analog to digital

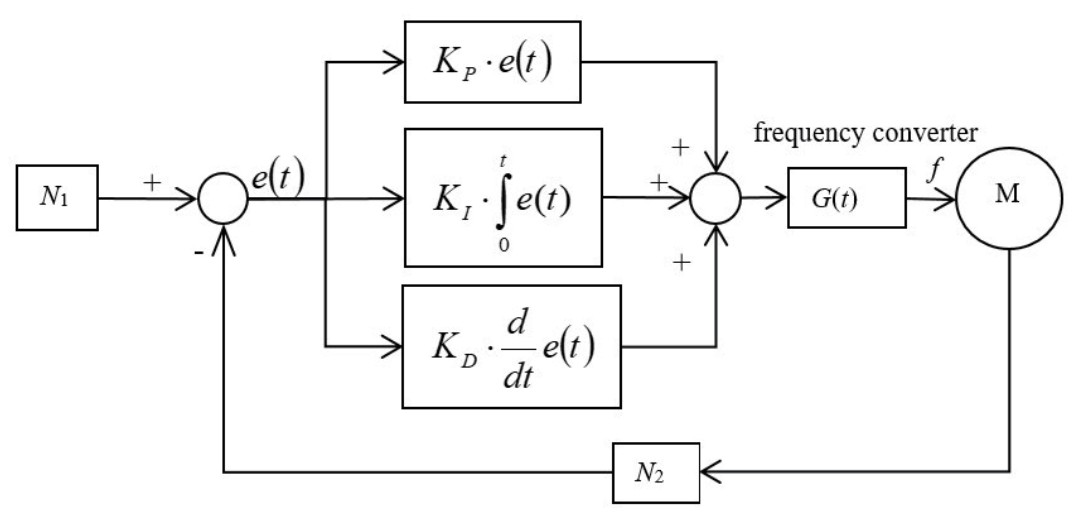

Fig. 5. Control loop of AVS method.

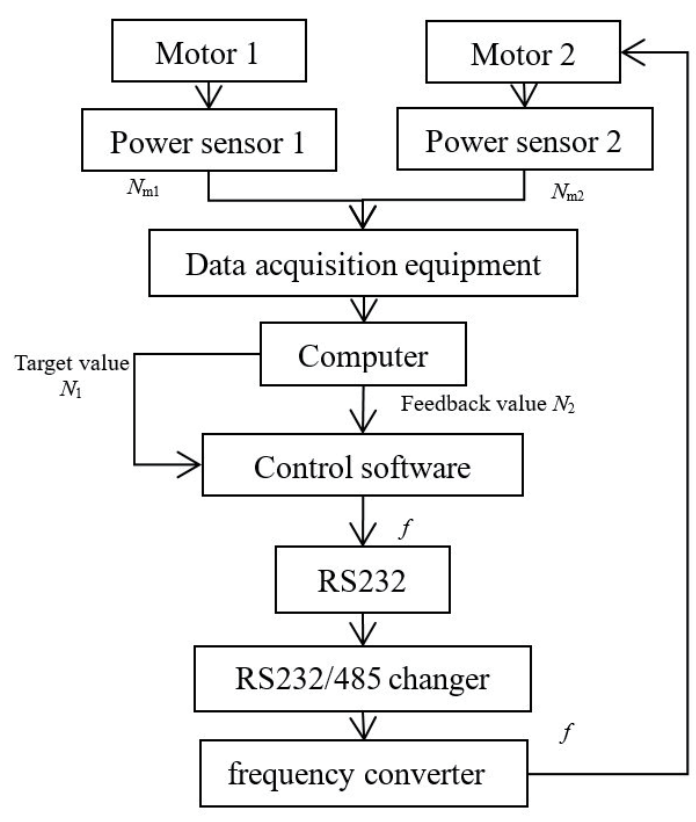

Fig. 6. Control process of AVS. 
via an $\mathrm{A} / \mathrm{D}$ converter and then sent to a $\mathrm{CPU}$ unit for further calculations. In real time, we calculate the success rate and $\Delta N$. Initially, we set a threshold value of the first-level error, denoted as $\varepsilon$. When $-\varepsilon<\Delta N<\varepsilon$, which means that the deviation between the input powers of R1 and R2 is small and can be neglected, the speed of R2 is kept unchanged. On the other hand, when $\Delta N<-\varepsilon$, we increase the speed of $\mathrm{R} 2$ to a suitable value by sending a control signal through an RS232 serial port and a frequency converter to a CPU unit, then check the value of $\Delta N$ and continuously tune it until the powers of $\mathrm{R} 1$ and $\mathrm{R} 2$ are equal. When $\Delta N>\varepsilon$, we decrease the speed of $\mathrm{R} 2$ to a suitable value in the same way.

In experiments, the adopted data acquisition board has a measurement range of $0-+10000 \mathrm{mV}$. The adopted power sensor is a power transmitter (model NB-AP3B1-A6EC) with a measurement range of $0-10 \mathrm{~kW}$ and an output voltage range of $1-5 \mathrm{~V}$ (transmitted to a data acquisition board). We use a model CVF-G2/P2 frequency converter (Bosch Rexroth Co., Ltd.) to control the speed of the rotors, which is connected to the calculator via an RS232/485 converter.

\subsubsection{Control process of AVS method}

The speed control process for R2 in the AVS method is performed on a LabVIEW platform. The AVS control mainly includes two parts: (1) power signal collection and calculations; (2) speed control using frequency converters. Figure 7 shows the calculation procedure of the computation strategy in the frequency converter. First, we collect signals detected by power sensors via a data acquisition board. Second, we manipulate the acquired data using the

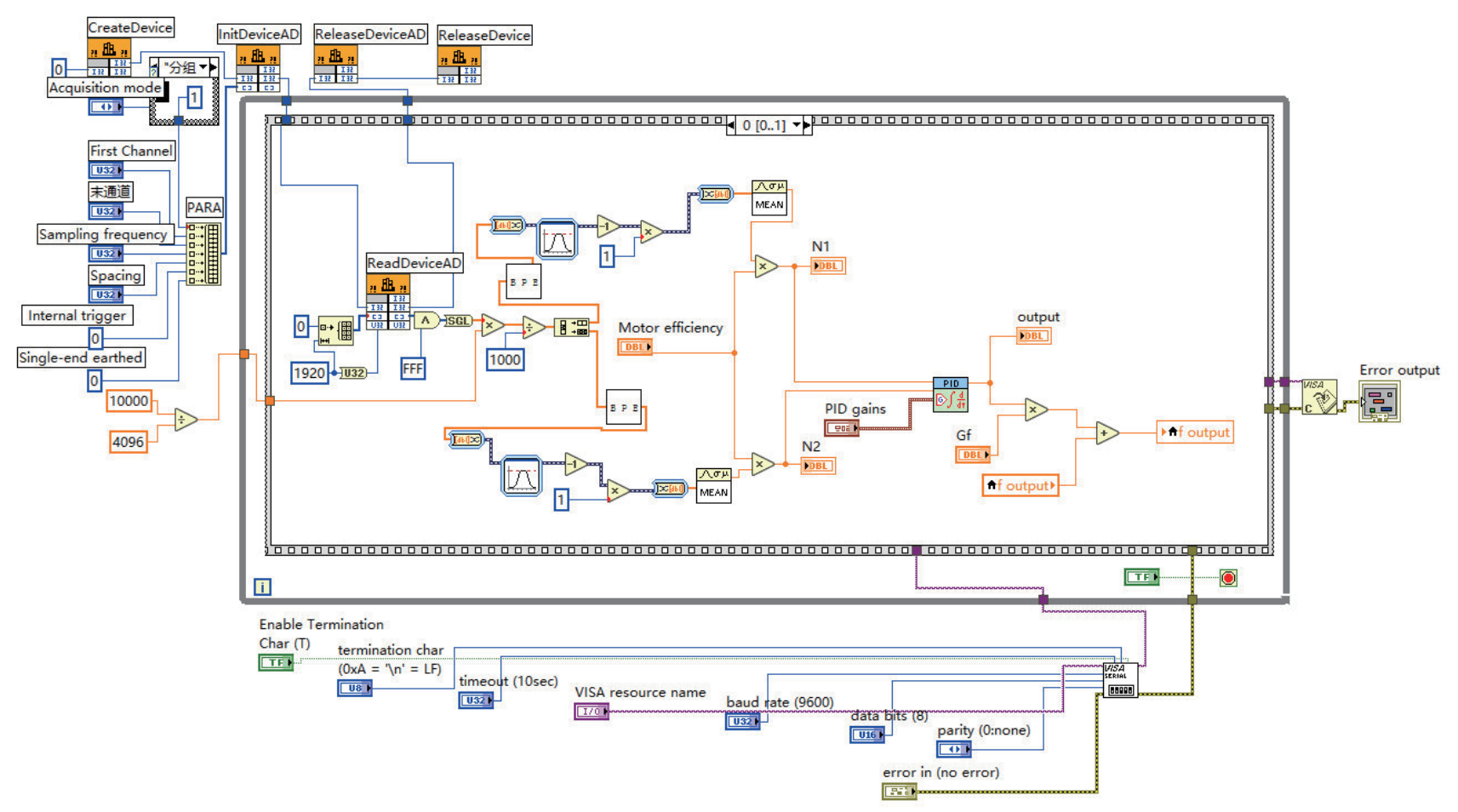

Fig. 7. (Color online) Main calculation procedure of computation strategy in frequency converter. 
singularity replacement and digital filtering process. Finally, the final power outputs of R1 and R2 are obtained. According to the proposed control process shown in Fig. 6 and the output power relationship between the two rotors, we may suitably adjust the input current of R2 in real time.

Our proposed AVS method adopts the so-called Pauta criterion ( $3 \sigma$ principle). The following is the manipulation procedure. Note that during the power measurement process for the two rotors, the elimination replacement method is used to handle the singularity that occurs. Firstly, we independently measure the power of every rotor $n$ times with an equal-precision method and obtain the data series $\left\{x_{1}, x_{2}, \ldots, x_{n}\right\}$. The absolute errors of the obtained data are defined as $d_{1}=x_{1}-\bar{x}, d_{2}=x_{2}-\bar{x}, \ldots, d_{n}=x_{n}-\bar{x}$, where $\bar{x}$ is the average value. If the data measured at a certain point, $x_{k}$, has an absolute error of $\left|d_{k}>3 \sigma\right|$ ( $\sigma$ : standard deviation of measured data), then the point with the value of $x_{k}$ may be viewed as a singular point and is replaced by

$$
x_{k}=\left\{\begin{array}{ll}
\bar{x} & (k=1) \\
x_{k-1} & (k \neq 1)
\end{array}\right. \text {. }
$$

The process of singular value replacement is further programmed as a subroutine called VI of the main program of the AVS method. This subroutine, which is mainly used to increase the test precision of the whole MCRF system, is shown in Fig. 8.

Next, we develop a novel real-time control process for R2. As shown in Fig. 9, this process adopts protocols between the master and slave communications to fulfil series connection between the calculator and the frequency converter. The calculator is used as the master unit and the frequency converter as the slave unit. According to the communication protocols, the master unit sends orders and messages to the slave unit, and then the slave executes the corresponding actions. The data format of the master unit is hexadecimal, which is the format accepted by the frequency converter.

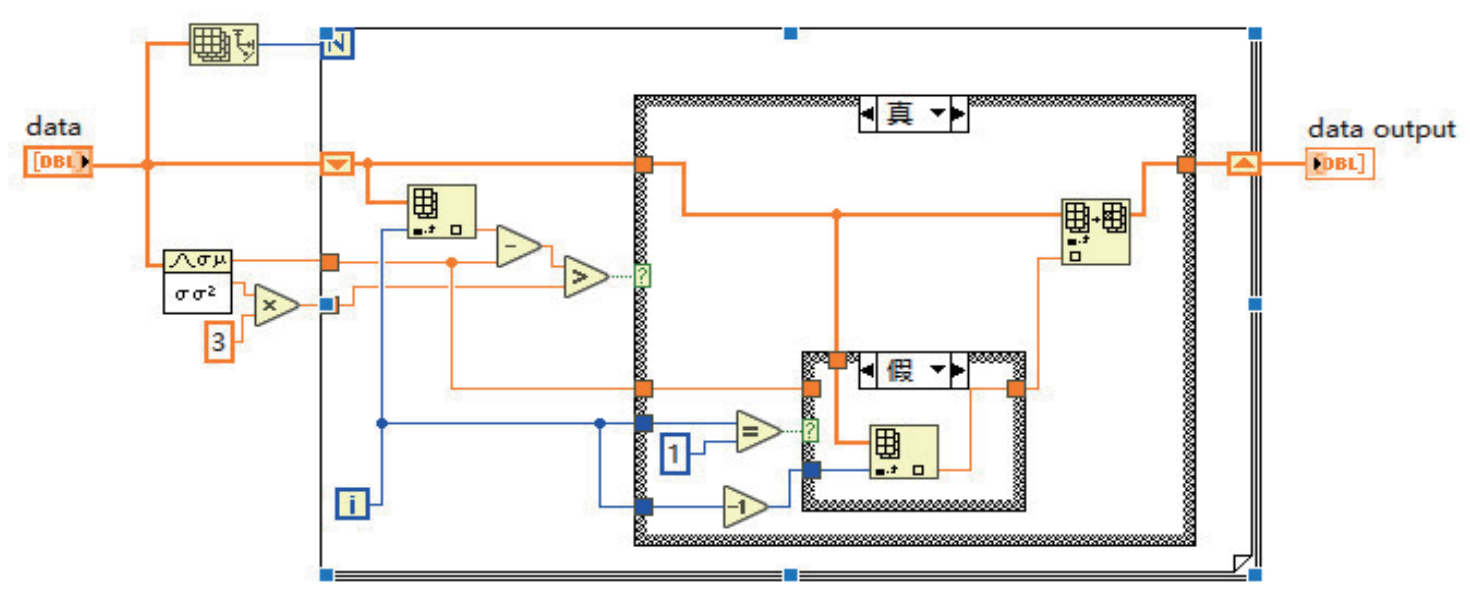

Fig. 8. (Color online) Subroutine VI for singular value replacement. 


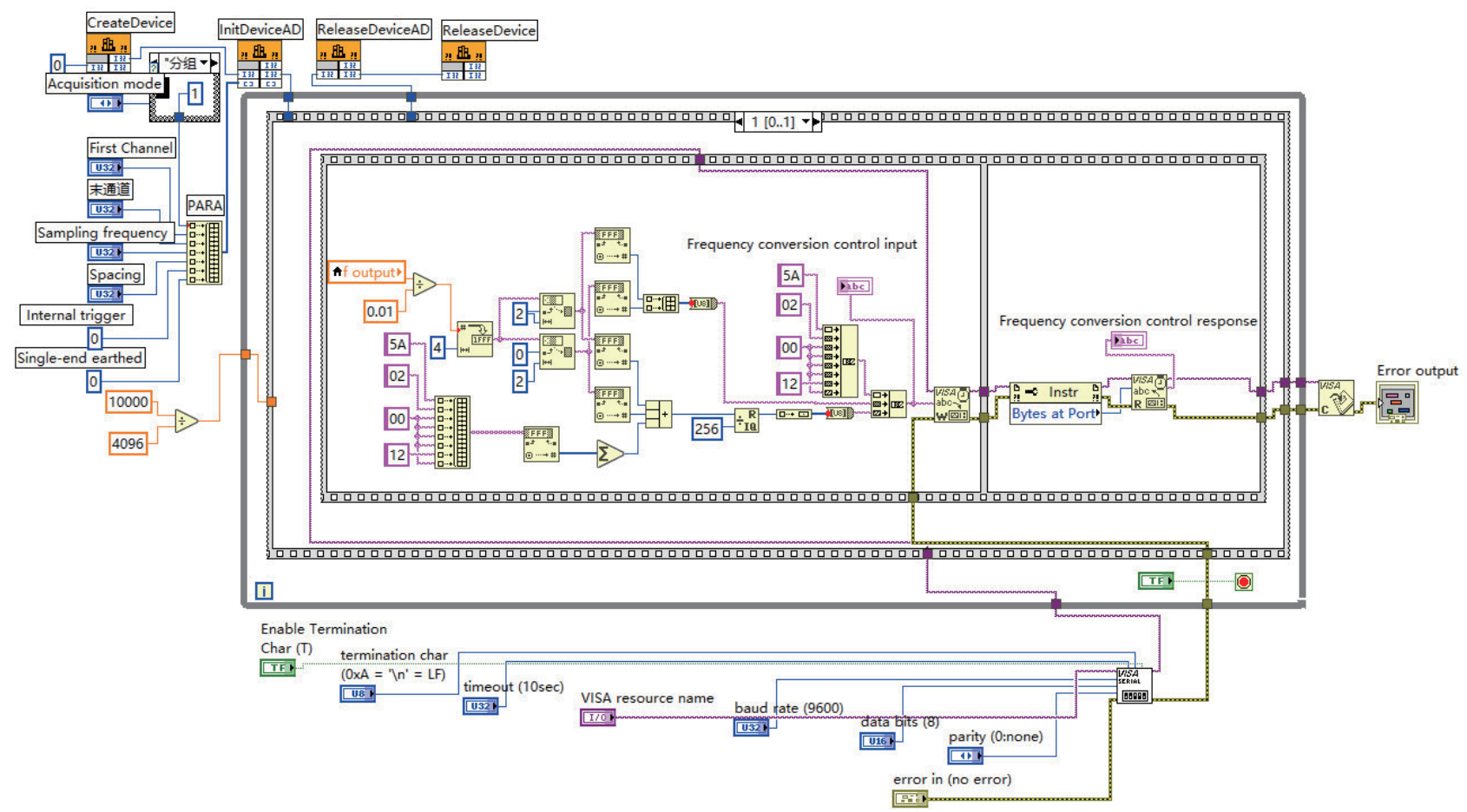

Fig. 9. (Color online) Real-time control process of R2.

\section{Results and Verification}

To test our proposed AVS control method, we carry out an experiment with the measurement instruments including various sensors shown in Fig. 2. The experiment is designed to adaptively adjust the speed of the rotors in our designed MCRF system in real time by changing the resistance force at the inlet pipe net while simultaneously measuring the related gas-dynamic characteristics of the fan system. The measured power and pressure variations with the mass flow rate of the MCRF system are shown in Fig. 10. Compared with the measured results without AVS control shown in Figs. 3 and 4, the performance of the whole MCRF system with AVS control is much higher. The MCRF system without AVS control has low performance because R1 and R2 are always kept at the same operation speed and cannot be individually adjusted in real time with changes in mass flow rate. However, the MCRF system with AVS control may adaptively adjust the power output of R2 according to changes in mass flow rate. In detail, through AVS control, the power of R2 may be automatically and suitably increased and decreased with increasing and decreasing mass flow rate, respectively. Consequently, in the whole working range, R1 and R2 can always be kept at the same speed, and thus the variation of pressure with mass flow becomes smooth and the blocking condition shifts to the right. Moreover, the overload problem of R2, which occurs when the mass flow rate suddenly drops, can be solved. Furthermore, with this AVS control method, the overall efficiency of the MCRF system and the working range both increase. 

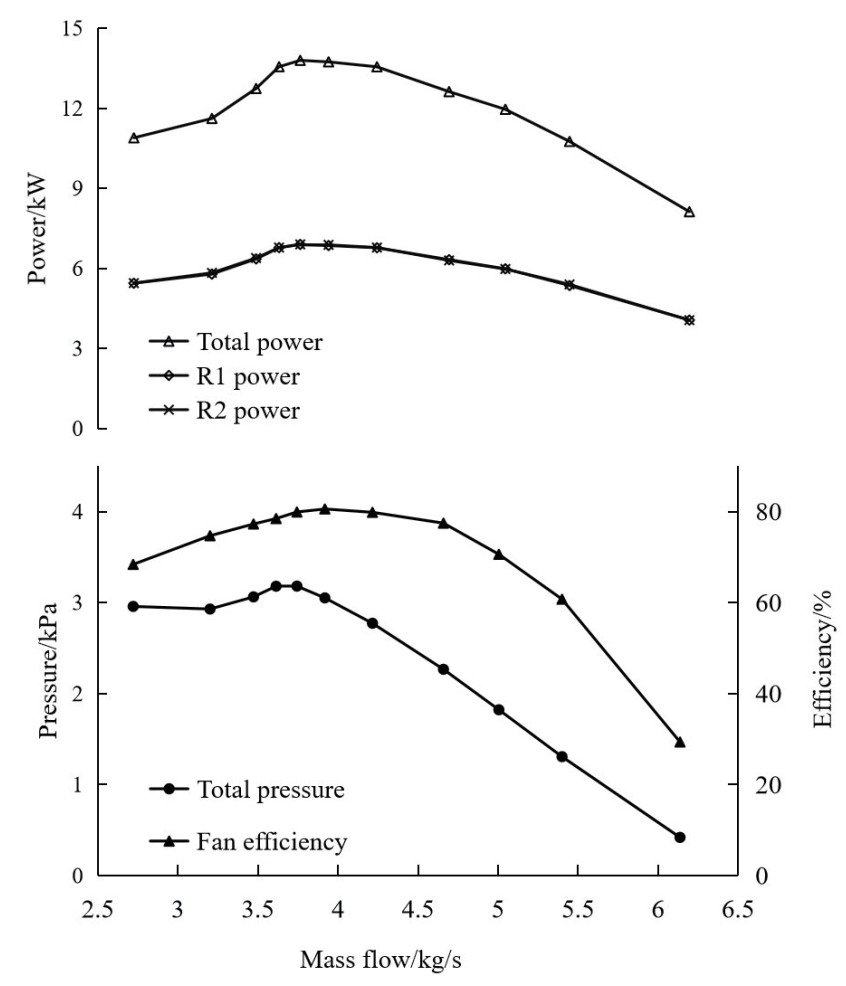

Fig. 10. Performance of MCRF system using AVS control.

For an MCRF system, the blocking margin is defined as

$$
\text { blocking margin }=\left(q_{\max }-q_{\text {des }}\right) / q_{\text {des }} \times 100 \% \text {, }
$$

where $q_{\max }(\mathrm{kg} / \mathrm{s})$ is the maximum mass flow rate under the blocking condition and $q_{\text {des }}(\mathrm{kg} / \mathrm{s})$ is the rated or designed flow rate. The working range of an MCRF system operating under a highefficiency condition is defined as

$$
\text { working range }=q_{90} / q_{d e s} \times 100 \% \text {, }
$$

where $q_{90}(\mathrm{~kg} / \mathrm{s})$ is the mass flow rate whose rated efficiency is greater than or equal to $90 \%$ under stable working conditions. The blocking conditions before and after AVS control for our designed MCRF system are shown in Fig. 11. The rated mass flow rate is $q_{d e s}=4.25 \mathrm{~kg} / \mathrm{s}$, the maximum mass flow rate under the blocking condition in the equal-speed operation of R1 and $\mathrm{R} 2$ is $q_{\max }=5.64 \mathrm{~kg} / \mathrm{s}$, and the maximum mass flow rate at the blocking margin under AVS control is increased to $q_{\max }=6.14 \mathrm{~kg} / \mathrm{s}$. By using Eq. (1), we obtain a blocking margin of $32.71 \%$ for the MCRF system in the case of equal-speed operation of R1 and R2 without AVS control and a blocking margin of $44.47 \%$ with AVS control, an increase of about $36 \%$. The adoption of AVS control clearly increases the working range of the MCRF system.

Figure 12 shows the results of the working range under the high-efficiency condition before and after AVS control for our designed MCRF system. It can be seen that the threshold mass 


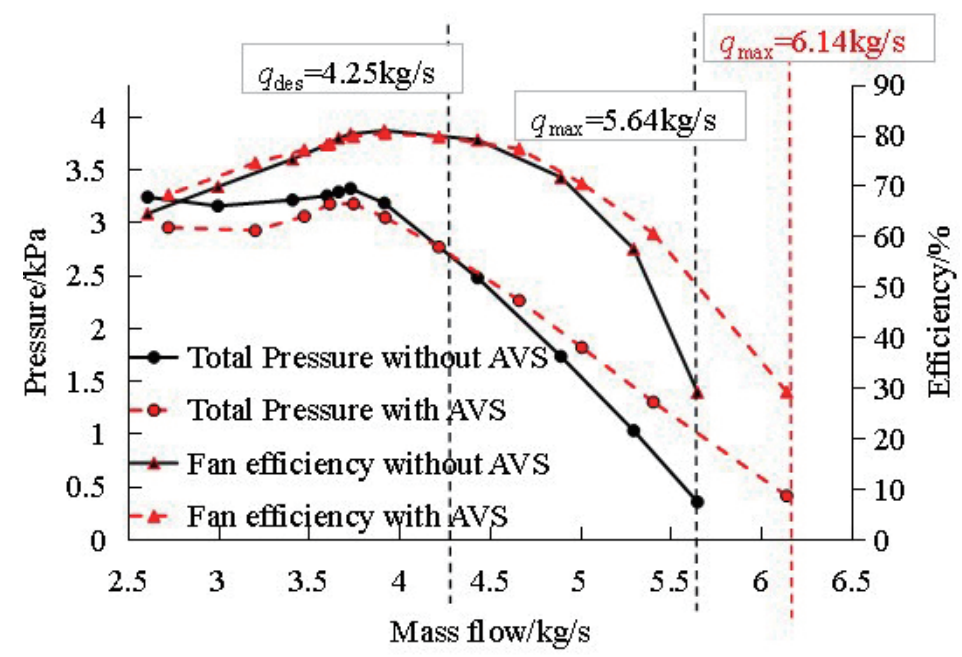

Fig. 11. (Color online) Blocking conditions before and after AVS control.

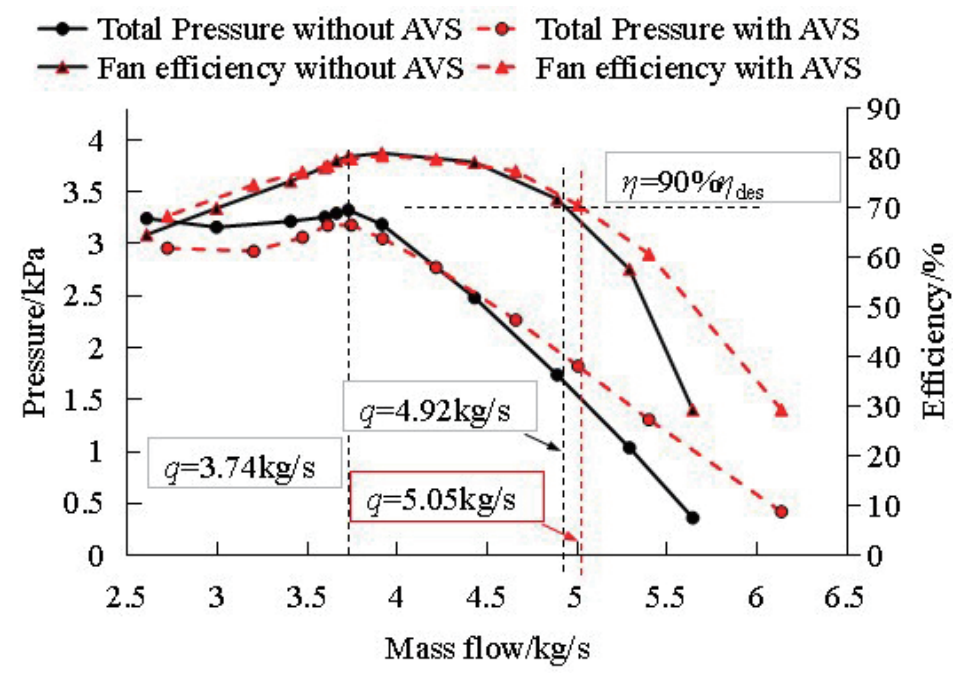

Fig. 12. (Color online) Results of the working range under high-efficiency condition before and after AVS control for our designed MCRF system.

flow rate between the stable and unstable working conditions is $q_{d i v}=3.74 \mathrm{~kg} / \mathrm{s}$. For the equalspeed operation of R1 and R2, the working mass flow ranges under a high-efficiency condition are $q_{90}=4.92-3.74=1.18 \mathrm{~kg} / \mathrm{s}$ before AVS control and $q_{90}=5.05-3.74=1.31 \mathrm{~kg} / \mathrm{s}$ after AVS control. From Eq. (3), we determine the working range under high-efficiency operation to be $27.76 \%$ under the equal-speed operation of R1 and R2, which increases to $30.82 \%$ under AVS control. Consequently, the working range of our designed MCRF with AVS control is expanded.

In summary, to solve the overload problem, we adopted the traditional method with different loads (e.g., by reducing the load) or raised the power margin for R2. ${ }^{(25-28)}$ By comparing our proposed method of AVS control with traditional methods, we observed that our novel method is more comprehensive and has higher performance. 


\section{Conclusion}

We propose an AVS control method to improve the overall performance of an MCRF system. The novelty of the method is that it may adaptively control the speed of the second-stage rotor (R2) according to changes in the working conditions of the whole fan system. In addition, we have also performed some experiments with various sensors to detect the pressure and mass flow rate for our newly designed MCRF system. We found that without AVS control, the performance of R1 remained stable with high efficiency within the whole working range, while that of R2 deteriorated and overload occurred under a small mass flow rate and the performance markedly decreased under a large mass flow rate. These behaviors significantly limited the performance as well as the working range under high-efficiency operation. Also, problems of operation safety arose. With AVS control, the speed of R2 may be adaptively changed in real time according to changes in the working conditions of the whole fan system. The experimental results illustrate that through AVS control, the working performance of R2 as well as the overall MCRF system can be increased. The problem of overload under a small mass flow rate can be avoided. Furthermore, the working range with high efficiency is enlarged and the blocking margin is increased. Consequently, the overall working range of the MCRF system is also enlarged.

\section{Acknowledgments}

This research was supported by the Natural Science Foundation of Fujian Province (grant number 2019J01822) and the National Natural Science Foundation of China (grant number 52176043).

\section{References}

1 L. Yueze, S. Akhtar, A. P. Sasmito, and J. C. Kurnia: Int. J. Min. Sci. Technol. 27 (2017) 657. https://doi. org/10.1016/j.ijmst.2017.05.019

2 M. Rezaeian and H. Montazeri: Technol. Forecast. Soc. Change 118 (2017) 270. https://doi.org/10.1016/j. techfore.2017.02.027

3 F. Geng, G. Luo, Y. Wang, Z. Peng, S. Hu, T. Zhang, and H. Chai: Process Saf. Environ. Prot. 113 (2018) 388. https://doi.org/10.1016/j.psep.2017.11.010

4 H. Nouri, F. Ravelet, F. Bakir, and R. Rey: J. Fluids Eng. 134 (2012) 104504. https://doi.org/10.1115/1.4007591

5 C. Friebe, O. Velde, R. Krause, and K. Hackeschmidt: FAN2018 Darmstadt (Germany) 2018 (2018) 18.

6 P. B. Sharma, D. S. Pundhir, and K. K. Chaudhry: J. Def. Model. Simul. 41 (2013) 165. https://doi.org/10.14429/ dsj.41.4424

7 C. Mistry and A. M. Pradeep: ASME J. Turbomach. 136 (2014) 071009. https://doi.org/10.1115/1.4025953

8 T. Shigemitsu, Y. Takeshima, Y. Ogawa, and J. Fukutomi: IOP Conf. Ser.: Earth Environ. 49 (2016) 102008. https://doi.org/10.1088/1755-1315/49/10/102008

9 T. D. Toge and A. M. Pradeep: Aerosp. Sci. Technol. 70 (2017) 534. https://doi.org/10.1016/j.ast.2017.08.041

10 B. J. Lee, M. F. Liou, and M. L. Celestina: NASA Technology Reports Server, ISABE-2019-24285 (2019) 1. Corpus ID: 209372149.

11 X. Zhang and Y. Wang: Int. J. Aeronaut. Space Sci. 20 (2019) 100. https://doi.org/10.1007/s42405-018-0108-1

12 H. Luan, L. Weng, R. Liu, D. Li, and M. Wang: Int. J. Aerosp. Eng. 2019 (2019) 1. https://doi. org $/ 10.1155 / 2019 / 2125976$

13 T. Shigemitsu, K. Miyazaki, K. Hirosawa, and H. Fukoda: Int. J. Comput. Fluid Dyn. 8 (2018) 181. https://doi. org/10.4236/ojfd.2018.82013 
14 M. J. Kingan and A. B. Parry: J. Fluid Mech. 868 (2019) 385. https://doi.org/10.1017/jfm.2019.172

15 X. Mao, B. Liu, and H. Zhao: Proc. Inst. Mech. Eng., Part G: J. Aerosp. Eng. 233 (2019). https://doi. org/10.1177/0954410017745901

16 Y. Wang, W. Chen, C. Wu, and S. Ren: Proc. Inst. Mech. Eng., Part G: J. Aerosp. Eng. 229 (2015) 1953. https:// doi.org/10.1177/0954410014562483

17 B. Liu, X. C. Mao, P. Zhang, H. Chen, and X. X. Wu: J. Propul. Power 2016 (2016) 1. https://doi.org/10.13675/j. cnki.tjjs.2016.05.003

18 X. Mao, B. Liu B, and H. Zhao: Proc. Inst. Mech. Eng., Part G: J. Aerosp. Eng. 233 (2019) 1059. https://doi. org/10.1177/0954410017745901

19 W. Chen, Y. Wang, and H. Wang: J. Therm. Sci. 28 (2019) 962. https://doi.org/10.1007/s11630-019-1191-8

20 H. Luan, L. Weng, Y. Luan, Y. Zhang, and P. Chen: J. Vib. Eng. Technol. 18 (2016) 5605. https://doi. org/10.21595/jve.2016.17926

21 C. Mistry and A. M. Pradeep: Proc. Inst. Mech. Eng., Part A: J. Power Energy 227 (2013) 138. https://doi. org/10.1177/0957650912467453

22 H. Luan, L. Weng, and Y. Luan: PLOS ONE 13 (2018) e0200510. https://doi.org/10.1371/journal.pone.0200510

23 H. Luan, L. Weng, R. Liu, and D. Li: Int. J. Aerosp. Eng. 2019 (2019) 1. https://doi.org/10.1155/2019/2125976

24 B. Dong, C. Jiang, X. Liu, Y. Deng, and L. Huang: Mech. Syst. Sig. Process. 135 (2020) 106362. https://doi. org/10.1016/j.ymssp.2019.106362

25 M. J. Shahriyari, H. Khaleghi, and M. Heinrich: J. Eng. Gas Turbines Power 141 (2019) 081009. https://doi. org/10.1115/1.4043251

26 C. Mistry and A. M. Pradeep: Propul. Power Res. 3 (2014) 68. https://doi.org/10.1016/j.jppr.2014.05.005

27 D. W. Meng, Y. Y. Xia, and Q. Wang: IEEE Int. Conf. Applied Superconductivity \& Electromagnetic Devices 2015 (2015) 15936394. https://doi.org/10.1109/ASEMD.2015.7453471

28 F. Ravelet, F. Bakir, C. Sarraf, and J. Wang: Exp. Therm Fluid Sci. 96 (2018) S0894177718303297. https://hal. archives-ouvertes.fr/hal-01610997

29 X. Sun, D. Meng, B. Liu, and Q. Wang: Adv. Mech. Eng. 9 (2017) 1687814017720083. https://doi. org $/ 10.1177 / 1687814017720083$

30 D. W. Meng, X. Sun, and Z. Zhang: 2015 2nd Int. Conf. Machinery, Materials Engineering, Chemical Engineering and Biotechnology (2015). https://doi.org/10.2991/mmeceb-15.2016.190

31 C. Huo, P. Lv, and A. Sun: Int. J. Micro Air Veh. 11 (2019) 1756829319833686. https://doi. org $/ 10.1177 / 1756829319833686$ 